\title{
Lecture \\ Helping another in distress: Lessons from rats
}

\author{
PEGGY MASON ${ }^{11}$
}

\begin{abstract}
The basis of affectively motivated helping of another in distress has long been debated by scholars in diverse disciplines. Work in rodents that took place more than 50 years ago suggested that rodents participate in affective communication. Now the author's laboratory has established an ethical and feasible test for rodent helping behavior that involves one rat freeing another from a plastic tube. The helping exhibited is consistent, reinforced, socially selective, and independent of immediate social contact. A recent modified version of the helping behavior test confirms that rats help a conspecific in need. In sum, the complex social behavior, expressed by rodents and primates including humans, validates the notion that mammals share a phylogenetic inheritance that promotes other-oriented affective behavior.
\end{abstract}

Key words : Empathy, helping, pro-social behavior, altruism, rodents

In 2008, I was deep into my studies on the mechanisms by which morphine and other opioids affect bulbospinal neurons to alter nociceptive processing in the dorsal horn. Spikes and milliseconds constituted my focus. My goal was to understand how neurons in the medulla determine whether noxious stimulation elicits either a perception of pain or not (aka analgesia) depending on the circumstances. One of those circumstances was opioid administration.

Not being of a pharmacological bent, I carved out a scientific niche for my laboratory by looking at nociceptive modulation during species-specific behaviors (Mason, 2005). It was remarkable, as well as teleologically satisfying, to find that ethological behaviors - sleeping, micturition, eating are accompanied by analgesia, albeit in short bouts. For example, during the seconds

1) Department of Neurobiology, University of Chicago, Chicago, IL

947 East $58^{\text {th }}$ St. MC 0926, Chicago, IL 60637, USA

Corresponding author: PEGGY MASON

E-mail: pmason@uchicago.edu

J-STAGE Advance Published Date: November 13, 2015. doi: 10.2502/janip.65.2.1 required to empty the bladder, a rat is insensitive to noxious stimulation (Baez, Brink \& Mason, 2006). He does not respond. He does not withdraw although during periods of urine storage, the rat readily withdraws from application of the same stimulus.

Even as I focused on brainstem modulation of pain, I knew that what is popularly referred to as "psychology" could also influence pain perception as powerfully as morphine does. The placebo effect is a good example (Levine, Gordon, Bornstein, \& Fields, 1979; Wager, Rilling, Smith, Sokolik, Casey, Davidson, Kosslyn, Rose, \& Cohen, 2004). Another good example is empathy, which can lead to feelings of pain in the absence of any stimulus. Work by many, including Tania Singer and Jean Decety, demonstrated that watching another receive a noxious stimulus can elicit an empathic experience of pain in the viewer (Bernhardt \& Singer, 2012; Jackson, Meltzoff, \& Decety, 2005; Lamm, Batson, \& Decety, 2007; Lamm, Decety, \& Singer, 2011; Singer, Seymour, O'Doherty, Kaube, Dolan, \& Frith, 2004). Moreover, vicariously experienced pain is accompanied by activation in regions of the brain 
overlapping with those activated when a person directly experiences stimulus-evoked pain. Conversely, feeling understood and cared for by a health professional has nearly the same effect as morphine on a patient's satisfaction (Dawson, Spross, Jablonski, Hoyer, Sellers, \& Solomon, M. Z. 2002).

Most neuroscientists, the author included, do not view psychology as anything more than really, really complicated brain circuits. I therefore jumped at the chance to collaborate with Jean Decety and Inbal Ben-Ami Bartal in testing for evidence of empathy in rats. Specifically the idea was to test whether rats exposed to a conspecific in distress would respond by helping. If rats help out, possibly out of empathy, then helping is biological. The alternative would be that rats do not help others, which would support the notion that helping is a culturally taught, and therefore largely human, phenomenon. Two caveats are worth mentioning here. First, culture is simply an exponential of psychology, meaning that it is the very complex manifestation of combining many superbly complicated brain circuits. Second, as developed below, the answer to this question was fairly apparent from the existing literature. However, before continuing on, a definition of terms is in order.

\section{What is empathy? How do we define helping?}

Empathy has been defined in a myriad of ways across the large number of disciplines attracted to its study (Batson, 2009). Here we consider two definitions popular within the neuroscience and psychology communities. At the root of both definitions is the communication of affect between individuals. In other words, any alteration in the behavior or state of one individual caused by the affect of another is considered empathy by those favoring an "umbrella definition" of empathy. A more restrictive definition requires that affective sharing be other-oriented in order to constitute empathy (Bernhardt \& Singer, 2012). According to this latter definition, catching sadness or joy from another would not constitute empathy unless one understood that the sadness or joy originated in the other. For example, viewing a fearful individual would need to engender concern for that individual rather than concern for the self in order to constitute empathy. This requirement for knowing that the other is the source of the feeling is a high bar for rodents. More accurately it is a high bar for those of us studying rodents. Several years of working in this field have revealed the difficulties of unequivocally demonstrating the internal state of a rodent.

Here I employ the umbrella term of empathy, viz. affective or emotional communication between individuals. This definition is neutral in the sense that empathy may fuel selfish behavior, helping (an otheroriented positive action), or even cruelty or schadenfreude (an other-oriented negative action). The term empathic concern is used to refer to other-oriented empathy, a definition that replaces the emotional neutrality of empathy with a requirement for pro-sociality. Empathic concern can motivate intentional actions that benefit another. Such actions are termed helping or pro-social behavior.

Let's unpack our definition of helping. Benefiting another inadvertently does not constitute helping. The helper has to intend that his or her actions will benefit the other. Yet, there is no requirement that the other be the only one to reap benefits from a helping action. A helping action is still considered helping even if it benefits the self (the helper) as well as the other (the recipient of help). The benefit to the self may be internal. Indeed, humans experience the "warm glow of helping" when donating to charity, whether by choice or mandate (Harbaugh, Mayr, \& Burghart, 2007). While such an internal feeling may appear to provide miniscule benefit, the brain regions that are activated when humans help by giving money to others are the same areas that are critical to reinforcing drug-taking (Moll, Krueger, Zahn, Pardini, de Oliveira-Souza, \& Grafman, 2006). These results suggest that entirely selfless giving (sometimes termed altruism) is not biologically realistic. Inten- 
tional actions require reinforcement. Reinforcement is associated with a positive affect whereas an action that is consistently accompanied by a neutral or negative affective experience is unlikely to be reinforced. In sum, helping is an intentional action that benefits the other, typically with a coincident benefit to the self.

\section{Emotional contagion in rodents}

Early studies on empathic helping were revolutionary as they introduced the notion that motivated pro-social behavior, which was widely assumed to be restricted to primates, was amenable to laboratory investigations employing rodents (Church, 1959; Rice \& Gainer, 1962). Yet the models employed in these early studies had methodological and ethical limitations that have limited acceptance of the studies' conclusions and precluded use of the paradigms used.

In the past decade or so, there have been numerous demonstrations that mice and rats show emotional contagion, a rudimentary form of empathy (Chen, Panksepp, \& Lahvis, 2009; Jeon, Kim, Chetana, Jo, Ruley, Lin, Rabah, Kinet, \& Shin, 2010 Langford, Crager, Shehzad, Smith, Sotocinal, Levenstadt, Chanda, Levitin, \& Mogil, 2006; Panskepp \& Lahvis, 2011; Panskepp \& Panskepp, 2013; Watanabe, 2011, 2012). For example, a mouse may exhibit fear in response to a conditioning stimulus (CS) without ever directly experiencing pairing of that $\mathrm{CS}$ with an unconditioned stimulus (UCS; Panskepp \& Lahvis, 2011). In these experiments, mice acquired a fear response to the CS from a social interaction with another mouse that directly experienced CS-UCS pairing. A model of reciprocal helping has been reported from one laboratory (Rutte \& Taborsky, 2007). In sum, there have been several recent demonstrations that affective communication between individual rodents occurs.

\section{Helping in rodents}

Building upon the above reports of emotional contagion in rodents, we were interested in whether rats would express pro-social behavior if they were given the opportunity to do so. In other words, if helping could be accomplished through a simple action, an action within the behavioral repertoire of a rodent, would a rat help another rat in distress? Here I describe the experiments, results, and interpretation that lead us to answer that question in the affirmative.

In the behavioral helping test, one rat is placed within a transparent Plexiglas tube, or restrainer, that is centrally located in a testing arena (Ben-Ami Bartal, Decety, \& Mason, 2011). A second rat is able to roam free throughout the arena. The door to the restrainer can only be opened from the outside and therefore only by the free rat. Building on a wealth of evidence that rodents experience restraint as a stressor (Glavin, Paré, Sandbak, Bakke, \& Murison, 1994), the focus of the helping test is on the free rat's reaction to the trapped rat's distress. In essence, the behavioral helping test offers the opportunity for one rat to end another rat's distress. Rat pairs are tested for 12 daily sessions of an hour each.

Will the free rat open the door to the restrainer and liberate the trapped rat? As it turns out, the answer is yes. Free rats begin to consistently open the restrainer door as early as the first or second day of testing (Ben-Ami Bartal, Decety, \& Mason, 2011). Moreover, a rat that opens on one day is extremely likely to open on the following day (Fig. 1). Thus, even without receiving any external reward, most rats repeatedly venture into the arena center and open a restrainer door. Rats who open on sequential days at least three times, termed openers, constitute about $75 \%$ of all rats tested with cagemates. Rats open the restrainer door for a trapped rat but not for an empty restrainer or a restrainer filled with a toy rat, demonstrating that a trapped rat is necessary for eliciting door-opening from the free rat.

Opening the restrainer door is socially selective in that rats will only open for rats from familiar stocks, evidence that the free rat is motivated to open by particular features of the trapped rat (Ben-Ami Bartal, 
Rodgers, Bernardez Sarria, Decety, \& Mason, 2014). We have not yet identified the crucial features. Yet, the finding of socially selective door-opening supports the importance of a trapped rat to motivating door-opening. Further, this selectivity enabled us to manipulate experimental conditions in order to study a large number of non-openers as well as openers. As shown in Figure 2, openers nearly always open on sequential days, whereas non-openers almost never do. This dichotomous difference shows that the definition of opener rats is meaningful. Openers do not haphazardly meet an arbitrary criterion and then quit opening. Rather, opener rats handily surpass the criteria. These findings are strong evidence that dooropening is highly reinforcing.

The evidence presented so far shows that door-opening is 1) reinforcing; 2) dependent on the presence of a trapped rat; and 3) only

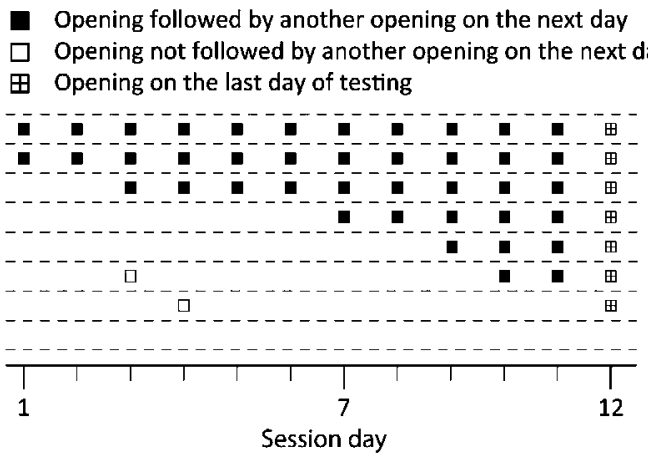

Figure 1. A summary of the opening behavior of a typical cohort of 8 male Sprague-Dawley rat pairs is shown. Openings that were not followed by another opening on the subsequent day are symbolized by an open square. Openings that were followed by another opening on the subsequent day are symbolized by a filled square. For openings on the last testing day (cross-filled squares), it is unclear whether the rats would have opened on the following day. All pairs had been cagemates for two weeks prior to the beginning of habituation and testing. Free rats in the top 6 pairs are considered "openers" as they open on consecutive days at least three times. Free rats in the bottom two pairs are considered "non-openers" as they did not meet the above criteria. All data are from the $S D$ cagemate group reported in Ben-Ami Bartal, Rodgers, Bernardez Sarria, et al. (2014). extended to rats of a familiar stock. These features are consistent with door-opening being an act of helping. Yet the simplest explanation for door-opening is a desire to play with the trapped rat. To test this idea, we placed two arenas together and put the restrainer up against the divide so that opening the door would release the trapped rat into the second arena (Ben-Ami Bartal, Decety, \& Mason, 2011). Using this approach, the free rat was prevented from accessing his cagemate once he opened the door to the restrainer. The erstwhile trapped rat was released into a separate space from that of the free rat. We tested 9 openers in this "separated" condition. Rats were tested with a trapped cagemate or with an empty restrainer in counterbalanced order. We found that when there was a rat in the restrainer, free rats opened the door, even though they could not play with the beneficiary of their

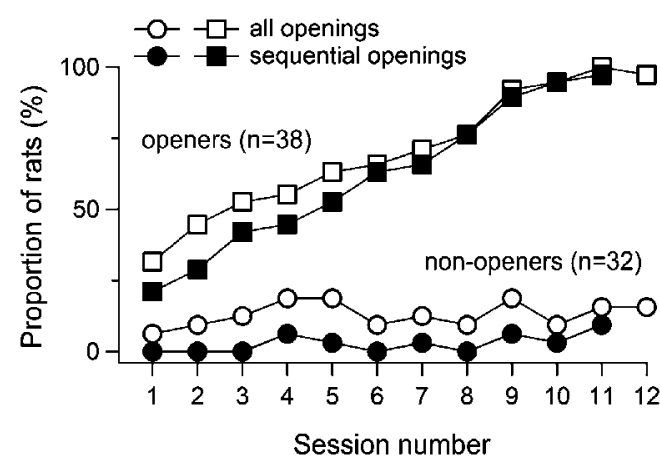

Figure 2. The proportion of sequential openings relative to all openings is shown for 70 male Sprague-Dawley rat pairs tested with trapped male rats. Trapped rats were from SpragueDawley or Long Evans stocks, cagemates or strangers, and from a familiar or unfamiliar stock. Openings that were not followed by another opening on the subsequent day are represented by open symbols. Openings that were followed by another opening on the subsequent day are represented by filled symbols. Note that a sequential opening was not possible on the final testing day. Another opening on the next day followed almost all door-openings performed by openers. In contrast, the number of openings by non-openers was low and the number of consecutive openings even lower. These data have been reported previously in Ben-Ami Bartal, Rodgers, Bernardez Sarria, et al. (2014). 
action. When the rat was tested with an empty restrainer in the separated setup, door-opening behavior extinguished. These results demonstrate that rats engage in prosocial behavior even when prevented from immediate social contact.

It is important to fully consider the meaning of the result that immediate social contact is not needed to motivate dooropening. This result simply means that the immediate, most proximal motivation for door-opening does not involve social interaction. The result does not in any way imply that social reward is unimportant. Ultimately, social reward is at the basis of all social behaviors.

Despite our experiment showing that immediate social contact is not needed to motivate door-opening, the idea that the rats in our study were motivated by social reward persisted. This idea received support from a perplexing study that purported to repeat our experimental design but in fact bore no resemblance to our study (Silberberg, Allouch, Sandfort, Kearns, Karpel, \& Slotnick, 2014). The authors set up a constantly changing set of rules for the rats that is challenging even for human readers to understand. Fundamentally, it is unclear what the authors were testing. No evidence is presented to demonstrate that the rats understood the experimental contingencies. The simplest explanation for the Silberberg, Allouch, Sandfort, et al. (2014) results is that since rats were re-trapped after each door-opening, the rats learned that opening the door was futile and simply opted for physical proximity.

\section{Confirmation that rats help}

At the 2014 meeting of the Japanese Society for Animal Psychology, I had the delightful experience of meeting Nobuya Sato and learning of his work on helping in rats. Dr. Sato subsequently published his study that confirms our work and extends it in interesting ways (Sato, Tan, Tate, \& Okada, 2015). The basic paradigm was to place one rat in a pool next to an arena with a potential helper rat. The "soaked" rat in the pool could only access the dry area if the other rat opened a circular door separating the two chambers. If the helper rat opened the door, the soaked rat could climb onto a dry floor. What happened? Well, by the eighth day, the door was opened for the soaked rat in nine out of ten (five male, five female) cagemate pairs tested. Moreover, by the final day of testing, help arrived within about 20 seconds on average. Rats took 3-4 times longer to open the door when the pool was empty (water but no rat), dry (no water and no rat), or contained a toy object (no water and no rat but with a toy). Thus, rats open the door to help a cagemate reach a dry space and do so consistently at a shorter and shorter latency with every passing day.

The most appealing aspect of Dr. Sato's experimental paradigm is the ability to disambiguate space from distress. In other words, the pool occupied a sizable area and if it were not for the water, the area would be an acceptable place to be. Thus the space where the rat is trapped is not inherently stressful. In a critical experiment, rats were placed in adjoining areas without water that were separated by the circular door. If the test rat were motivated by the desire for social contact, then the rat would open the door between the two spaces. On the other hand, if the rat were motivated by affect, then the rat would not open the door. Why would the rat "help" when there's nothing wrong? As it turns out, the rats did not open the door. Thus rats only opened the door when they detected distress from the soaked rat. Or to put it another way, distress is needed to elicit help. This is a compelling demonstration that rats are motivated by the affect of another rat and tells us that the rat's action is best described as help rather than as a desire for social contact. In sum, Dr. Sato's work supports the finding that rats are motivated to help another in distress and places another nail in the coffin of the rats-open-for-social-contact idea.

\section{The value of helping}

Fundamentally, the finding of pro-social 
behavior from rodents demonstrates that helping another in distress is biologically rather than culturally based. It suggests that mammals have a biological pull to help. Culture, personal stress, or physical abilities may interfere with actual helping. Nonetheless, as the saying, to give is to get, highlights; helping clearly has a value to the helper as well as to the one who is helped. What is the value of helping? When rats were given a choice between opening a restrainer containing a trapped cagemate, one containing chocolate chips, or both, they consistently chose to open both restrainers (Ben-Ami Bartal, Decety, \& Mason, 2011). Moreover, there was no difference in the latency to open the two restrainers, suggesting that helping a cagemate has a value on par with chocolate.

Sato, Tan, Tate, and Okada, (2015) further explored the value of helping. Rats were placed in the middle of three chambers. They were trained with only two of the chambers used so that half were trained to open for chocolate and half for a rat in a pool. Then the rats were exposed to both chocolate and a rat in a pool, and given the choice of which door to open first. Those trained with the soaked rat opened first for the rat $80 \%$ of the time. But what was surprising was that those trained with chocolate opened first for the rat almost half the time. This is in contrast to the 20\% opening rate that would be expected if chocolate held the same sway as a cagemate in distress. The attraction of helping has great drawing power!! These results suggest that helping a cagemate has pulling power that chocolate does not, evidence that helping a cagemate may be more valuable than chocolate.

\section{Conclusions}

There are limits to what rats and the helping behavior test can tell us about humans. To understand these limits, let us imagine that helping occurs within a multidimensional space. Axes within this space include the cost of helping, the severity of the need, and the familiarity of the individual in need of help. Helping may or may not occur depending on where a given situation sits within the space. For example, a child asking for money to see a movie is far more likely to be helped than if a stranger asks for money to see the same film. On the other hand, when the need for help appears lifethreatening, the requirement for familiarity is tossed out the window. The news is replete with stories of heroic individuals who help an injured stranger in a time of acute crisis.

Despite enormous differences, humans and rats may be fundamentally alike, sharing similar forms of social behavior inherited through a common phylogenetic history. As mammals, both species are capable of detecting and responding to benefit distressed others. Humans often do this using language but rats use actions that move physical objects in the world. Although popular culture suggests that a chasm separates saying and doing, this idea is not supported by neurobiology. The muscles of speech laryngeal, abdominal and upper airway muscles - are skeletal muscles that require input from motoneurons just as the biceps, quadriceps, pectoral muscles are. In essence, speaking and writing are no more and no less actions than are lifting a weight and opening a door. There is nothing more (or less) accurate or honest about an action using laryngeal muscles than one using limb muscles. With this motor equivalence in mind, rats' actions may speak just as loudly as humans' actions do. Given the fractious state of modern society, we may do well to listen to the lessons that rats can teach us.

\section{References}

Baez, M. A., Brink, T. S., \& Mason, P. 2005 Roles for pain modulatory cells during micturition and continence. Journal of Neuroscience, 25(2), 384-394.

Batson, C. D. 2009 These things called empathy. In J. Decety, W. Ickes (Eds.), The Social Neuroscience of Empathy. Cambridge, MA: MIT Press. Pp.16-31. 
Ben-Ami Bartal, I., Decety, J., \& Mason, P. 2011 Empathy and pro-social behavior in rats. Science, 334(6061), 1427-1430.

Ben-Ami Bartal, I., Rodgers, D. A., Bernardez Sarria, M. S., Decety, J., \& Mason, P. 2014 Pro-social behavior in rats is modulated by social experience. eLife, 3 , e01385.

Bernhardt, B. C., \& Singer, T. 2012 The neural basis of empathy. Annual Review of Neuroscience, 35, 1-23.

Chen, Q., Panksepp, J. B., \& Lahvis, G. P. 2009 Empathy is moderated by genetic background in mice. PLoS One, 4(2), e4387.

Church, R. M. 1959 Emotional reactions of rats to the pain of others. Journal of Comparative Physiology \& Psychology, 52(2), 132-134.

Dawson, R., Spross, J, A., Jablonski, E. S., Hoyer, D. R., Sellers, D. E., \& Solomon, M. Z. 2002 Probing the paradox of patients' satisfaction with inadequate pain management. Journal of Pain Symptom Management, 23(3), 211-220.

Glavin, G. B., Paré, W. P., Sandbak, T., Bakke, H. K., \& Murison, R. 1994 Restraint stress in biomedical research: an update. Neuroscience Biobehavioral Reviews, 18(2), 223-249.

Harbaugh, W. T., Mayr, U., \& Burghart, D. R. 2007 Neural responses to taxation and voluntary giving reveal motives for charitable donations. Science, 316(5831), 1622-1625.

Jackson, P. L., Meltzoff, A. N., \& Decety, J. 2005 How do we perceive the pain of others? A window into the neural processes involved in empathy. Neuroimage, 24(3), 771-779.

Jeon, D., Kim, S., Chetana, M., Jo, D., Ruley, H. E., Lin, S. Y., Rabah, D., Kinet, J. P., \& Shin, H. S. 2010 Observational fear learning involves affective pain system and Cav1.2 Ca2+ channels in ACC. Nature Neuroscience, 13(4), 482-488.

Lamm, C., Batson, C. D., \& Decety, J. 2007 The neural substrate of human empathy: effects of perspective-taking and cognitive appraisal. Journal of Cognitive Neuroscience, 19(1), 42-58.

Lamm, C., Decety, J., \& Singer, T. 2011 Metaanalytic evidence forcommonand distinct neural networks associated with directly experienced pain and empathy for pain. Neuroimage, 54(3), 2492-2502.

Langford, D. J., Crager, S. E., Shehzad, Z., Smith, S. B., Sotocinal, S. G., Levenstadt, J. S., Chanda, M. L., Levitin, D. J., \& Mogil, J. S. 2006 Social modulation of pain as evidence for empathy in mice. Science, 312(5782), 1967-1970.

Levine, J. D., Gordon, N. C., Bornstein, J. C., \& Fields, H. L. 1979 Role of pain in placebo analgesia. Proceedings of the National Academy of Sciences, 76(7), 35283531.

Mason, P. 2005 Ventromedial medulla: pain modulation and beyond. Journal of Com parative Neurology, 493(1), 2-8.

Moll, J., Krueger, F., Zahn, R., Pardini, M., de Oliveira-Souza, R., \& Grafman, J. 2006 Human fronto-mesolimbic networks guide decisions about charitable donation. Proceedings of the National Academy of Sciences, 103(42), 15623-15628.

Panksepp, J., \& Panksepp, J. B. 2013 Toward a cross-species understanding of empathy. Trends in Neuroscience, 36(8), 489-496.

Panksepp, J. B., \& Lahvis, G. P. 2011 Rodent empathy and affective neuroscience. Neu roscience Biobehavioral Reviews, 35(9), 18641875.

Rice, G. E., \& Gainer, P. 1962 "Altruism" in the albino rat. Journal of Comparative Physiology \& Psychology, 55, 123-125.

Rutte, C., \& Taborsky, M. 2007 Generalized reciprocity in rats. PLoS Biology, 5(7), e196.

Sato, N., Tan, L., Tate, K., \& Okada, M. 2015 Rats demonstrate helping behavior toward a soaked conspecific. Animal Cognition, 18(5), 1039-1047.

Silberberg, A., Allouch, C., Sandfort, S., Kearns, D., Karpel, H., \& Slotnick, B. 2014 Desire for social contact, not empathy, may explain "rescue" behavior in rats. Animal Cognition, 17(3), 609-618. 
Singer, T., Seymour, B., O'Doherty, J., Kaube, H., Dolan, R. J., \& Frith, C. D. 2004 Empathy for pain involves the affective but not sensory components of pain. Science, 303(5661), 1157-1162.

Wager, T. D., Rilling, J. K., Smith, E. E., Sokolik, A., Casey, K. L., Davidson, R. J., Kosslyn, S. M., Rose, R. M., \& Cohen, J. D. 2004 Placebo-induced changes in FMRI in the anticipation and experience of pain.
Science, 303(5661), 1162-1167.

Watanabe, S. 2011 Empathy and reversed empathy of stress in mice. PLoS One, 6 (8), e23357.

Watanabe, S. 2012 Distress of mice induces approach behavior but has an aversive property for conspecifics. Behavioral Processes, 90(2), 167-73.

(2015. 9.27 受稿, 2015.10. 8 受理) 